\title{
ON THE OPERATIONAL DETERMINATION OF TWO DIMENSIONAL GREEN'S FUNCTIONS IN THE THEORY OF HEAT CONDUCTION $\dagger$
}

\author{
A. N. LOWAN
}

1. Introduction. In a previous paper $\ddagger$ the writer has described an operational method for evaluating Green's functions in the theory of heat conduction and illustrated the method for the case of a semiinfinite solid. In this case the starting point was the solution of the differential equation of heat conduction satisfying the condition of a plane source.

It is the object of this paper to illustrate the same method for the case of the two dimensional flow of heat, in which the starting point is the solution of the differential equation of heat conduction satisfying the condition for a line source.

Specifically, we shall determine the Green's functions for the cases where the solid is one of the two following:

(A) An infinite cylinder.

(B) A solid bounded internally by a cylinder.

In both cases we shall take the boundary condition in the form

$$
\frac{\partial u}{\partial r}+h u=0 \quad \text { for } \quad r=a .
$$

From the general solution to be derived it will be easy, by making $h=0$ or $h=\infty$ in the general solution, $\S$ to obtain the corresponding solutions for the two important cases where the boundary is (1) impervious to heat, (2) kept at $0^{\circ}$.

2. Case (A). We start with the solution

$$
u\left(r, \theta, t ; r_{0}, \theta_{0}\right)=\frac{1}{4 \pi k t} \exp \left\{-\frac{r^{2}+r_{0}^{2}-2 r r_{0} \cos \left(\theta-\theta_{0}\right)}{4 k t}\right\}
$$

which satisfies the condition for a line source at $\left(r_{0}, \theta_{0}\right)$. The solution (1) may be written in the equivalent forms

$\dagger$ Presented to the Society, October 30, 1937.

$\ddagger$ Philosophical Magazine, (7), vol. 24 (1937), pp. 62-70.

$\S$ Some special cases of the problems discussed in this paper have been treated by S. Goldstein, Proceedings of the London Mathematical Society, (2), vol. 34 (1932), pp. 51-88. Goldstein treats the case where the line source coincides with the axis of the cylinder. His boundary condition is $u=0$ or $\partial u / \partial r=0$. 


$$
u\left(r, \theta, t ; r_{0}, \theta_{0}\right)=\frac{1}{2 \pi} \int_{0}^{\infty} \alpha e^{-k \alpha^{2} t} J_{0}(R \alpha) d \alpha,
$$

where $R^{2}=r^{2}+r_{0}^{\dot{2}}-2 r r_{0} \cos \left(\theta-\theta_{0}\right)$, or

$\left(1^{\prime \prime}\right) u\left(r, \theta, t ; r_{0}, \theta_{0}\right)=\frac{1}{2 \pi} \sum_{n=-\infty}^{\infty} \cos n\left(\theta-\theta_{0}\right) \cdot \int_{0}^{\infty} \alpha e^{-k \alpha^{2} t} J_{n}(\alpha r) J_{n}\left(\alpha r_{0}\right) d \alpha$.

The Laplace transform of $\left(1^{\prime \prime}\right)$ is

$$
u^{*}\left(r, \theta, p ; r_{0}, \theta_{0}\right)=\int_{0}^{\infty} e^{-p t} u\left(r, \theta, t ; r_{0}, \theta_{0}\right) d t
$$

where $p$ is a complex parameter whose real part is positive. From ( $\left.1^{\prime \prime}\right)$ we get

$$
\begin{aligned}
u^{*}\left(r, \theta, p ; r_{0}, \theta_{0}\right)= & \frac{1}{2 \pi k} \sum_{n=-\infty}^{\infty} \cos n\left(\theta-\theta_{0}\right) \\
& \cdot \int_{0}^{\infty} \frac{\alpha}{\alpha^{2}-q^{2}} J_{n}(\alpha r) J_{n}\left(\alpha r_{0}\right) d \alpha
\end{aligned}
$$

where we have put $p=-k q^{2}$.

Consider the integral

$$
\begin{aligned}
S & =\int_{-\infty}^{\infty} \frac{\alpha}{\alpha^{2}-q^{2}} J_{n}(\alpha r) H_{n}{ }^{1}\left(\alpha r_{0}\right) d \alpha \\
& =\int_{-\infty}^{0} \frac{\alpha}{\alpha^{2}-q^{2}} J_{n}(\alpha r) H_{n}^{1}\left(\alpha r_{0}\right) d \alpha+\int_{0}^{\infty} \frac{\alpha}{\alpha^{2}-q^{2}} J_{n}(\alpha r) H_{n}{ }^{1}\left(\alpha r_{0}\right) d \alpha \\
& =S_{1}+S_{2} .
\end{aligned}
$$

In the first term make the substitution $\alpha=-\beta$. The integral becomes

$$
-\int_{0}^{\infty} \frac{\alpha}{\alpha^{2}-q^{2}} J_{n}(-\alpha r) H_{n}^{1}\left(-\alpha r_{0}\right) d \alpha
$$

where we have used $\alpha$ once more for the variable of integration. But

$$
J_{n}(-\alpha r)=(-1)^{n} J_{n}(\alpha r)
$$

and

$$
H_{n}^{1}\left(-\alpha r_{0}\right)=(-1)^{n}\left[H_{n}^{1}\left(\alpha r_{0}\right)-2 J_{n}\left(\alpha r_{0}\right)\right]
$$

Hence 
whence

$$
\begin{aligned}
S_{1} & =-\int_{0}^{\infty} \frac{\alpha d \alpha}{\alpha^{2}-q^{2}} J_{n}(\alpha r)\left[H_{n}^{1}\left(\alpha r_{0}\right)-2 J_{n}\left(\alpha r_{0}\right)\right] \\
& =-S_{2}+2 \int_{0}^{\infty} \frac{\alpha d \alpha}{\alpha^{2}-q^{2}} J_{n}(\alpha r) J_{n}\left(\alpha r_{0}\right),
\end{aligned}
$$

$$
\int_{0}^{\infty} \frac{\alpha d \alpha}{\alpha^{2}-q^{2}} J_{n}(\alpha r) J_{n}\left(\alpha r_{0}\right)=\frac{1}{2} \int_{-\infty}^{\infty} \frac{\alpha d \alpha}{\alpha^{2}-q^{2}} J_{n}(\alpha r) H_{n}^{1}\left(\alpha r_{0}\right) .
$$

The last identity evidently remains valid if we interchange $r$ and $r_{0}$.

Consider the complex integral

$$
\int_{C} \frac{\alpha d \alpha}{\alpha^{2}-q^{2}} J_{n}(\alpha r) H_{n}^{1}\left(\alpha r_{0}\right)
$$

in the case $r<r_{0}$, the path of integration $C$ consisting of the axis of reals and an infinite semicircle in the upper half of the $\alpha$-plane where the path is indented by a small semicircle around the origin. The contribution to the integral tends to zero as the radius of this semicircle tends to zero. From the known asymptotic behavior of $J_{n}$ and $H_{n}^{1}$ it is apparent that $\left|J_{n}(\alpha r) \cdot H_{n}^{1}\left(\alpha r_{0}\right)\right| \rightarrow 0$ as $|\alpha| \rightarrow \infty$. Thus the contribution to the integral from the infinite semicircle vanishes in the limit. Since the path $C$ contains the single pole $\alpha=q$, the application of Cauchy's theorem leads at once to the identity $\dagger$

$$
\int_{0}^{\infty} J_{n}\left(\alpha r_{0}\right) \cdot J_{n}(\alpha r) \frac{\alpha d \alpha}{\alpha^{2}-q^{2}}=\frac{\pi i}{2} J_{n}(r q) H_{n}{ }^{1}\left(r_{0} q\right), \quad r<r_{0} .
$$

In an entirely similar manner we obtain the identity

$$
\int_{0}^{\infty} J_{n}(\alpha r) \cdot J_{n}\left(\alpha r_{0}\right) \frac{\alpha d \alpha}{\alpha^{2}-q^{2}}=\frac{\pi i}{2} J_{n}\left(r_{0} q\right) \cdot H_{n}^{1}(r q), \quad r>r_{0} .
$$

With the aid of the identities (5) and $\left(5^{\prime}\right)$, (2) yields

(6) $u^{*}\left(r, \theta, p ; r_{0}, \theta_{0}\right)=\frac{i}{4 k} \sum_{n=-\infty}^{\infty} \cos n\left(\theta-\theta_{0}\right) J_{n}\left(r_{0} q\right) \cdot H_{n}^{1}(r q), \quad r>r_{0}$, and

$\left(6^{\prime}\right) u^{*}\left(r, \theta, p ; r_{0}, \theta_{0}\right)=\frac{i}{4 k} \sum_{n=-\infty}^{\infty} \cos n\left(\theta-\theta_{0}\right) \cdot J_{n}(r q) H_{n}{ }^{1}\left(r_{0} q\right), \quad r<r_{0}$.

$\dagger$ This is one of several identities obtained by Hankel, Mathematische Annalen, vol. 8 (1875), pp. 453-470, by integrating a more general integrand around the appropriate path. 
From (6) we get

$$
\begin{aligned}
\left(\frac{\partial u^{*}}{\partial r}+h u^{*}\right)_{r=a}=\frac{i}{4 k} & \sum_{n=-\infty}^{\infty} \cos n\left(\theta-\theta_{0}\right) \cdot J_{n}\left(r_{0} q\right) \\
& \cdot\left\{q \frac{d}{d z} H_{n}^{1}(z)+h H_{n}^{1}(z)\right\}_{z=a q} .
\end{aligned}
$$

In order to obtain the Green's function we must add to the line source solution $u$ a function $v$ satisfying the differential equation of heat conduction and vanishing at $t=0$. Its Laplace transform must then satisfy the differential equation

$$
\Delta v^{*}+q^{2} v^{*}=0,
$$

whence

$$
v^{*}=\frac{i}{4 k} \sum_{n=-\infty}^{\infty} A_{n} \cos n\left(\theta-\theta_{0}\right) J_{n}(q r)
$$

From (9) we get

$$
\begin{aligned}
& \left(\frac{\partial v^{*}}{\partial r}+h v^{*}\right)_{r=a} \\
& \quad=\frac{i}{4 k} \sum_{n=-\infty}^{\infty} A_{n} \cos n\left(\theta-\theta_{0}\right)\left\{q \frac{d}{d z} J_{n}(z)+h J_{n}(z)\right\}_{z=q a} .
\end{aligned}
$$

Since

$$
\left(\frac{\partial}{\partial r}+h\right)\left(u^{*}+v^{*}\right)=0 \quad \text { at } \quad r=a,
$$

it follows that

$$
A_{n}=-J_{n}\left(r_{0} q\right) \frac{q\left[\frac{d}{d z} H_{n}^{1}(z)\right]_{z=a q}+h H_{n}^{1}(a q)}{q J_{n}^{\prime}(a q)+h J_{n}(a q)}
$$

and therefore ultimately

$$
u^{*}+v^{*}=\frac{i}{4 k} \sum_{n=-\infty}^{\infty} \cos n\left(\theta-\theta_{0}\right) W_{n}^{*},
$$

where 


$$
\begin{aligned}
& p W_{n}^{*}=p \frac{J_{n}(q r)}{U_{n}(a q)}\left\{H_{n}^{1}\left(r_{0} q\right)\right. U_{n}(a q)-J_{n}\left(r_{0} q\right) \\
&\left.\cdot\left[\frac{z}{a} \frac{d}{d z} H_{n}^{1}(z)+h H_{n}^{1}(z)\right]_{z=a q}\right\}
\end{aligned}
$$

and $U_{n}(a q)=q J_{n}^{\prime}(a q)+h J_{n}(a q)$.

Remembering that $p=-k q^{2}$, we see that the expression $p W_{n}^{*}$ is of the form $Y(p) / Z(p)$. The transition from $p W_{n}^{*}$ to $W_{n}$ is equivalent to the inversion of the Laplace transform defining $W_{n}^{*}$, and we have

$$
W_{n}=\frac{Y(0)}{Z(0)}+\sum \frac{Y\left(p_{i}\right)}{p_{i} Z^{\prime}\left(p_{i}\right)} e^{p_{i} t},
$$

the summation being extended over the roots of $Z(p)=0$. We proceed to evaluate the second member of (15). The first term is evidently zero. Further

$$
Z(p)=Z\left(-k q^{2}\right)=U_{n}(a q)=q J_{n}^{\prime}(a q)+h J_{n}(a q),
$$

and therefore

$$
\begin{aligned}
\frac{d}{d p} Z(p) & =\frac{d U_{n}}{d q} \frac{d q}{d p}=-\frac{a}{2 k q}\left\{\frac{d}{d z}\left[\frac{z}{a} J_{n}^{\prime}(z)+h J_{n}(z)\right]\right\}_{z=a q} \\
& =\frac{-1}{2 k q}\left\{z J_{n}^{\prime \prime}(z)+(1+a h) J_{n}(z)\right\}_{z=a q} .
\end{aligned}
$$

But

$$
J_{n}^{\prime \prime}(z)+\frac{1}{z} J_{n}^{\prime}(z)+\left(1-\frac{n^{2}}{z^{2}}\right) J_{n}(z)=0,
$$

and from $Z(p)=U_{n}(a q)=0$ we get

$$
\frac{z}{a} J_{n}^{\prime}(z)+h J_{n}(z)=0 \quad \text { for } \quad z=a q .
$$

In view of (17) and (18), (16) becomes

$$
Z^{\prime}(p)=\frac{a}{2 k q^{2}}\left(h^{2}+q^{2}-\frac{n^{2}}{q^{2}}\right) J_{n}(a q) .
$$

In evaluating $Y\left(p_{i}\right)$ from (14) it is clear that the first term in brackets vanishes and thus 


$$
Y\left(p_{i}\right)=-p_{i} J_{n}\left(q_{i} r\right) J_{n}\left(q_{i} r_{0}\right)\left\{\frac{z}{a} \frac{d}{d z} H_{n}{ }^{1}(z)+h H_{n}{ }^{1}(z)\right\}_{z=a q_{i}} .
$$

If we make use of the identity

$$
J_{n}(z) \frac{d}{d z} H_{n}^{1}(z)-H_{n}^{1}(z) \frac{d}{d z} J_{n}(z)=\frac{2 i}{\pi z}
$$

and of (18), the expression in braces in (19) becomes

and therefore

$$
\frac{2 i}{\pi a J_{n}\left(q_{i} a\right)}
$$

$$
Y\left(p_{i}\right)=Y\left(-k q^{2}\right)=\frac{-2 i p_{i}}{\pi a} \frac{J_{n}\left(r q_{i}\right) J_{n}\left(r_{0} q_{i}\right)}{J_{n}\left(a q_{i}\right)} .
$$

With the aid of $(15),\left(16^{\prime}\right)$, and $\left(19^{\prime}\right)$ the inversion of (13) yields

$$
\begin{aligned}
G\left(r, \theta, t ; r_{0}, \theta_{0}\right)=u+v & =\frac{1}{\pi a^{2}} \sum_{n=-\infty}^{\infty} \cos n\left(\theta-\theta_{0}\right) \sum_{q_{i}} q_{i}{ }^{2} e^{-k q_{i}{ }^{2} t} \\
& \cdot \frac{J_{n}\left(q_{i} r\right) J_{n}\left(q_{i} r_{0}\right)}{\left(h^{2}+q_{i}{ }^{2}-\frac{n^{2}}{a^{2}}\right)\left\{J_{n}\left(q_{i} a\right)\right\}^{2}},
\end{aligned}
$$

where the second summation is extended over the roots of

$$
q J_{n}^{\prime}(a q)+h J_{n}(a q)=0 .
$$

From the general solution (22) we may obtain the solution for the case where the boundary is impervious to heat by putting $h=0$. Also the case where the boundary is kept at $0^{\circ}$ may be obtained by putting $h=\infty$. In this case it is clear that the transcendental equation (22) reduces to

$$
J_{n}(a q)=0 .
$$

Also it is easily seen that the denominator of (22) becomes $q^{2}\left\{J_{n}^{\prime}\left(q_{i} a\right)\right\}^{2}$.

Thus the Green's function for the case where the boundary is kept at $0^{\circ}$ is

(24) $G\left(r, \theta, t ; r_{0}, \theta_{0}\right)=\frac{1}{\pi a^{2}} \sum_{-\infty}^{\infty} \cos n\left(\theta-\theta_{0}\right) \sum_{q_{\boldsymbol{i}}} e^{-k q_{\boldsymbol{i}}{ }^{2 t}} \frac{J_{n}\left(q_{i} r\right) J_{n}\left(q_{i} r_{0}\right)}{\left\{J_{n}^{\prime}(q a)\right\}^{2}}$,

where the second summation extends over the roots of (23). 
3. Case (B). In this case, since the solution $v^{*}$ must be finite for $r=\infty$, and since $H_{n}^{1}(z) \rightarrow 0$ in the upper half of the z-plane, it follows that we must put

$$
v^{*}(r, \theta, p)=\frac{i}{4 k} \sum_{n=-\infty}^{\infty} A_{n} \cos n\left(\theta-\theta_{0}\right) \cdot H_{n}^{1}(q r) .
$$

From (25) we get

$$
\begin{aligned}
& \left(\frac{\partial v^{*}}{\partial r}+h v^{*}\right)_{r=a} \\
& \quad=\frac{i}{4 k} \sum A_{n} \cos n\left(\theta-\theta_{0}\right)\left\{q \frac{d}{d z} H_{n}^{1}(z)+h H_{n}^{1}(z)\right\}_{z=a q} .
\end{aligned}
$$

Also in this case, in view of (6) and $\left(6^{\prime}\right)$, we have

$$
\begin{aligned}
& \left(\frac{\partial u^{*}}{\partial r}+h u^{*}\right)_{r=a} \\
& \quad=\frac{i}{4 k} \sum \cos n\left(\theta-\theta_{0}\right) \cdot H_{n}^{1}\left(r_{0} q\right)\left\{q \frac{d}{d z} J_{n}(z)+h J_{n}(z)\right\}_{z=a q} .
\end{aligned}
$$

The condition

$$
\left(\frac{\partial}{\partial r}+h\right)\left(u^{*}+v^{*}\right)=0 \quad \text { for } \quad r=a
$$

thus yields ultimately

$$
u^{*}+v^{*}=\frac{i}{4 k} \sum_{n=-\infty}^{\infty} \cos n\left(\theta-\theta_{0}\right) W_{n}^{*},
$$

where

$$
\begin{aligned}
W_{n}^{*}= & J_{n}(r q) H_{n}^{1}\left(r_{0} q\right) \\
& -H_{n}^{1}\left(r_{0} q\right) \cdot H_{n}^{1}(r q)\left\{\frac{q \frac{d}{d z} J_{n}(z)+h J_{n}(z)}{q \frac{d}{d z} H_{n}^{1}(z)+h H_{n}^{1}(z)}\right\}_{z=a q} .
\end{aligned}
$$

Consider first the case $h=\infty$. In this case

$$
\begin{aligned}
W_{n}^{*} & =J_{n}(r q) H_{n}^{1}\left(r_{0} q\right)-\frac{J_{n}(a q)}{H_{n}{ }^{1}(a q)} \cdot H_{n}{ }^{1}\left(r_{0} q\right) \cdot H_{n}{ }^{1}(r q) \\
& =W_{n 1}^{*}+W_{n 2}^{*} .
\end{aligned}
$$


In view of (4) and (5) we have

$$
W_{n 1}^{*}=\frac{1}{\pi i} \int_{-\infty}^{\infty} \frac{\alpha}{\alpha^{2}-q^{2}} J_{n}(\alpha r) H_{n}^{1}\left(\alpha r_{0}\right) d \alpha
$$

It will be convenient to write $W_{n 2}^{*}$ in the form of a definite integral. For this purpose consider the integral

$$
\int_{C} \frac{\alpha}{\alpha^{2}-q^{2}} \frac{J_{n}(\alpha a)}{H_{n}^{1}(a \alpha)} \cdot H_{n}^{1}\left(r_{0} \alpha\right) H_{n}^{1}(r \alpha) d \alpha
$$

over the path $C$. From the asymptotic expansion of $J_{n}(z)$ and $H_{n}^{1}(z)$ it is easily seen that $H_{n}^{1}(r \alpha) / H_{n}^{1}(a \alpha)$ remains finite as $|\alpha| \rightarrow \infty$, and that $J_{n}(a \alpha) H_{n}^{1}\left(r_{0} \alpha\right) \rightarrow 0$ as $|\alpha| \rightarrow \infty$. Under these conditions the contribution from the large semicircle approaches zero as $|\alpha| \rightarrow \infty$. Furthermore the zeros of $H_{n}^{1}(z)$ are known to lie in the lower half of the $z$-plane. Thus the only pole of the integrand in the interior of $C$ is $\alpha=q$. Cauchy's theorem thus yields

$$
\begin{aligned}
W_{n 2}^{*} & =\frac{J_{n}(a q)}{H_{n}{ }^{1}(a q)} H_{n}^{1}(r q) H_{n}{ }^{1}\left(r_{0} q\right) \\
& =\frac{1}{\pi i} \int_{-\infty}^{\infty} \frac{\alpha}{\alpha^{2}-q_{2}} \frac{J_{n}(a \alpha)}{H_{n}^{1}(a \alpha)} H_{n}{ }^{1}(r \alpha) H_{n}{ }^{1}\left(r_{0} \alpha\right) d \alpha .
\end{aligned}
$$

In view of (32) and (33) we get

$$
W_{n}^{*}=\frac{1}{\pi i} \int_{-\infty}^{\infty} \frac{\alpha}{p+k \alpha^{2}}\left\{J_{n}(\alpha r) H_{n}{ }^{1}(\alpha a)-J_{n}(\alpha a) H_{n}{ }^{1}(\alpha r)\right\} \frac{H_{n}{ }^{1}\left(\alpha r_{0}\right)}{H_{n}{ }^{1}(\alpha a)} d \alpha,
$$

and therefore, finally,

$$
\begin{aligned}
u+v= & \frac{1}{4 \pi} \sum_{-\infty}^{\infty} \cos n\left(\theta-\theta_{0}\right) \cdot \int_{-\infty}^{\infty} \alpha e^{-k t \alpha^{2}} \\
& \cdot \frac{H_{n}{ }^{1}\left(\alpha r_{0}\right)}{H_{n}{ }^{1}(\alpha a)}\left\{J_{n}(\alpha r) \cdot H_{n}{ }^{1}(\alpha a)-J_{n}(\alpha a) H_{n}{ }^{1}(\alpha r)\right\} d \alpha .
\end{aligned}
$$

This is the solution of our problem when the cylindrical surface $r=a$ is kept at $0^{\circ}$.

Now consider the case where $h$ is finite. Then

$$
W_{n 2}^{*}=\left\{\frac{q \frac{d}{d z} J_{n}(z)+h J_{n}(z)}{q \frac{d}{d z} H_{n}^{1}(z)+h H_{n}^{1}(z)}\right\}_{z=a q} H_{n}^{1}(r q) H_{n}^{1}\left(r_{0} q\right) .
$$


Consider the integral

$$
\frac{1}{\pi i} \int_{C} \frac{\alpha}{\alpha^{2}-q^{2}}\left\{\frac{\alpha \frac{d}{d z} J_{n}(z)+h J_{n}(z)}{\alpha \frac{d}{d z} H_{n}^{1}(z)+h H_{n}^{1}(z)}\right\}_{z=a \alpha} H_{n}^{1}(r \alpha) H_{n}^{1}\left(r_{0} \alpha\right) d \alpha
$$

over the path $C$. As before it can be shown that the contribution from the large semicircle tends to zero as $|\alpha| \rightarrow \infty$. We now assume that the zeros of $q d H_{n}{ }^{1}(z) / d z+h H_{n}{ }^{1}(z)$ lie in the lower half plane. The Cauchy integral theorem leads to the identity

$\frac{1}{\pi i} \int_{-\infty}^{\infty} \frac{\alpha}{\alpha^{2}-q^{2}}\left\{\frac{\alpha \frac{d}{d z} J_{n}(z)+h J_{n}(z)}{\alpha \frac{d}{d z} H_{n}^{1}(z)+h H_{n}^{1}(z)}\right\}_{z=a \alpha} H_{n}^{1}(r \alpha) H_{n}^{1}\left(r_{0} \alpha\right) d \alpha=W_{n 2}^{*}$,

whence our final solution becomes

$$
\begin{aligned}
G=u+v= & \frac{1}{4 \pi} \sum_{n=-\infty}^{\infty} \cos n\left(\theta-\theta_{0}\right) \\
& \cdot \int_{-\infty}^{\infty} \alpha e^{-k \alpha^{2} t} \frac{H_{n}{ }^{1}\left(\alpha r_{0}\right)}{U_{n}(\alpha a)}\left\{J_{n}(\alpha r) U_{n}(\alpha a)-U_{n}(\alpha r) J_{n}(\alpha a)\right\} d \alpha,
\end{aligned}
$$

where

$$
U_{n}(\alpha a)=\left\{\alpha \frac{d}{d z} H_{n}^{1}(z)+h J_{n}(z)\right\}_{z=a \alpha} .
$$

For $h=\infty$ this solution yields our previous solution (21), as it should.

We have assumed above that there are no zeros of $U_{n}(a \alpha)$ in the interior of the path $C$. Briefly this can be shown by evaluating the integral

$$
\frac{1}{2 \pi i} \int_{C} \frac{\frac{d}{d z} U_{n}(z)}{U_{n}(z)} d z
$$

which, as is well known, represents the number of zeros of $U_{n}(z)$. Using the asymptotic expansion of $H_{n}^{1}(z)$, we find that the value of the above integral is equal to zero.

Brooklyn College

Yeshiva College 